The Astrophysical Journal, 216:L87-L89, 1977 September 15

(1) 1977. The American Astronomical Society. All rights reserved. Printed in U.S.A.

\title{
SPECTROSCOPY AND PHOTOMETRY OF THE DISTANT RADIO GALAXY 3C 343.1
}

\author{
HYRON SPINRAD \\ Department of Astronomy, University of California, Berkeley \\ AND \\ James Westphal, Jerome Kristian, and Allan Sandage \\ Hale Observatories, Carnegie Institution of Washington and California Institute of Technology \\ Received 1977 May 17; accepted 1977 June 17
}

\begin{abstract}
Spectrophotometry of the faint radio galaxy 3C 343.1 shows a strong emission line in the red. If identified with $\lambda 3727$ of [O II], it yields $z=0.750$. The identification is aided by noting that $\lambda 3869$ of $[\mathrm{Ne} \mathrm{III}]$ is marginally visible.

The optical continuum is relatively blue in color; it is likely to be caused by a population of stars younger than often found in nearby $\mathrm{E}$ galaxies. By comparison with other distant galaxies of established $z$, the isophotal diameter of about $7 "$, measured for $3 \mathrm{C} 343.1$, is consistent with the proposed redshift, and corresponds to a linear size of $70 \mathrm{kpc}$ to a surface brightness of $\mu_{r} \approx 26 \mathrm{mag} \mathrm{arcsec}^{-2}$. Subject headings: galaxies: redshifts - line identifications — radio sources: general
\end{abstract}

\section{INTRODUCTION AND OBSERVATIONS}

The objects 3C 343.1 and 3 C 343 are two adjacent $\left(\Delta=29^{\prime}\right)$ radio sources of approximately similar flux densities and spectral indices over the range 38-5000 $\mathrm{MHz}$ (Kellermann, Pauliny-Toth, and Williams 1969). They are both unresolved sources and are strong scintillators (Readhead and Hewish 1974).

However, Kristian and Sandage (1970) showed that the sources have very different optical counterparts: $3 \mathrm{C} 343$ is a $20.6 \mathrm{mag}$ quasar with $z=0.988$ (Baldwin et al. 1973), while 3C 343.1 is a faint galaxy, which we discuss here.

Figure 1 (Plate L2) shows the identification for 3C 343.1 on a new $4 \mathrm{~m}$ reflector plate; there are several other faint galaxies nearby (to the south), but it is unclear whether $3 \mathrm{C} 343.1$ is a cD galaxy in a BautzMorgan type I cluster, or an isolated, large radio galaxy.

Spectrophotometric observations with the Lick IDS system and the Hale 200 inch SIT vidicon and prism spectrograph both show a relatively narrow, strong emission line at $\lambda 6522$. If we categorically rule out blueshifts, then a single, fairly narrow emission line could only be $[\mathrm{O}$ II] $\lambda 3727$, or conceivably $\mathrm{L} \alpha$. Mg II emission (rare in galaxies) would be resolved into its doublet structure $(\Delta=13 \AA)$ on the Lick scans. Our experience with other radio galaxies indicates $\lambda 3727$ to be the overwhelming choice; thus $z=0.750$. On both the Hale and the Lick spectra there is weak evidence for the [Ne III] $\lambda 3869$ emission line. Figures 2 and 3 illustrate the Lick and Hale scans, respectively. Higher signal-to-noise ratios would be needed for us to be confident about the neon line. $\mathrm{H} \alpha$ at $\lambda=1.15 \mu \mathrm{m}$ should be observable with appropriate equipment.

\section{ISOPHOTAL DIAMETER AND PHOTOMETRY}

We measured the radial intensity profile of the galaxy 3C 343.1 from the KPNO $4 \mathrm{~m}$ plate (Fig. 1), using
Kron's interactive program on the Berkeley PDS. At a surface brightness of approximately $\mu_{r}=26 \mathrm{mag} \mathrm{arcsec}^{-2}$ the isophotal diameter is $\sim 7^{\prime \prime}$. We also list in Table 1 isophotal diameters $\theta_{s}$, and redshifts $z$, for other distant radio. galaxies for comparison. The individual values are still somewhat preliminary, and could contain systematic errors of up to $25 \%$ in $\theta_{s}$, but the $\theta, z$ pairs for 3C $295,3 \mathrm{C} 275$, and 3C 330 with 3C 343.1 clearly show a consistent pattern, lending support for our claim that $3 \mathrm{C} 343.1$ has a large redshift.

Diaphragm photoelectric photometry of both 3C 343 and $3 \mathrm{C} 343.1$ is tabulated in Table 2. The data were obtained on 1971 June 29 (UT), through an aperture of 7".6 with the Hale reflector. The colors for the $3 \mathrm{C} 343$ quasar are somewhat influenced by the strong emission lines of $\mathrm{Mg}$ II and [ $\mathrm{Ne} \mathrm{v]} \mathrm{(Baldwin} \mathrm{et} \mathrm{al.} \mathrm{1973).}$

We note that the radio galaxy $3 \mathrm{C} 343.1$ is quite blue compared to other distant radio and cluster $\mathrm{E}$ galaxies like 3C 295, 3C 330, and 3C 123. The blueness of 3C 343.1 continues out to at least $8000 \AA$ in the Lick scans, which yield $(R-I)_{\mathrm{sc}}=0.25 \pm 0.1 \mathrm{mag}$. This is much bluer than the M32 extrapolation to $z=0.75$ (cf. Spinrad 1976). The spectrophotometry shows no sign of the $\lambda_{0} 4000$ break, characteristic of the late-type stars whose spectra dominate most $\mathrm{E}$ galaxies. Unusual radio galaxy colors conventionally have two interpretations: (1) There might be a large contribution by stars hotter than the usual F5-G0 V turnoff seen in nearby ellipti-

TABLE 1

Some Radio Galaxy Isophotal Diameters

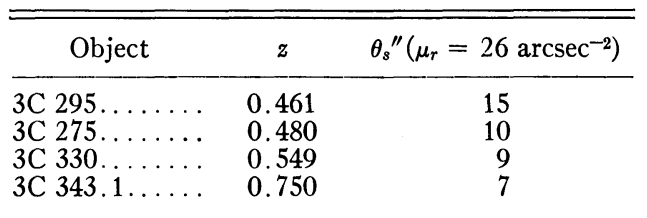




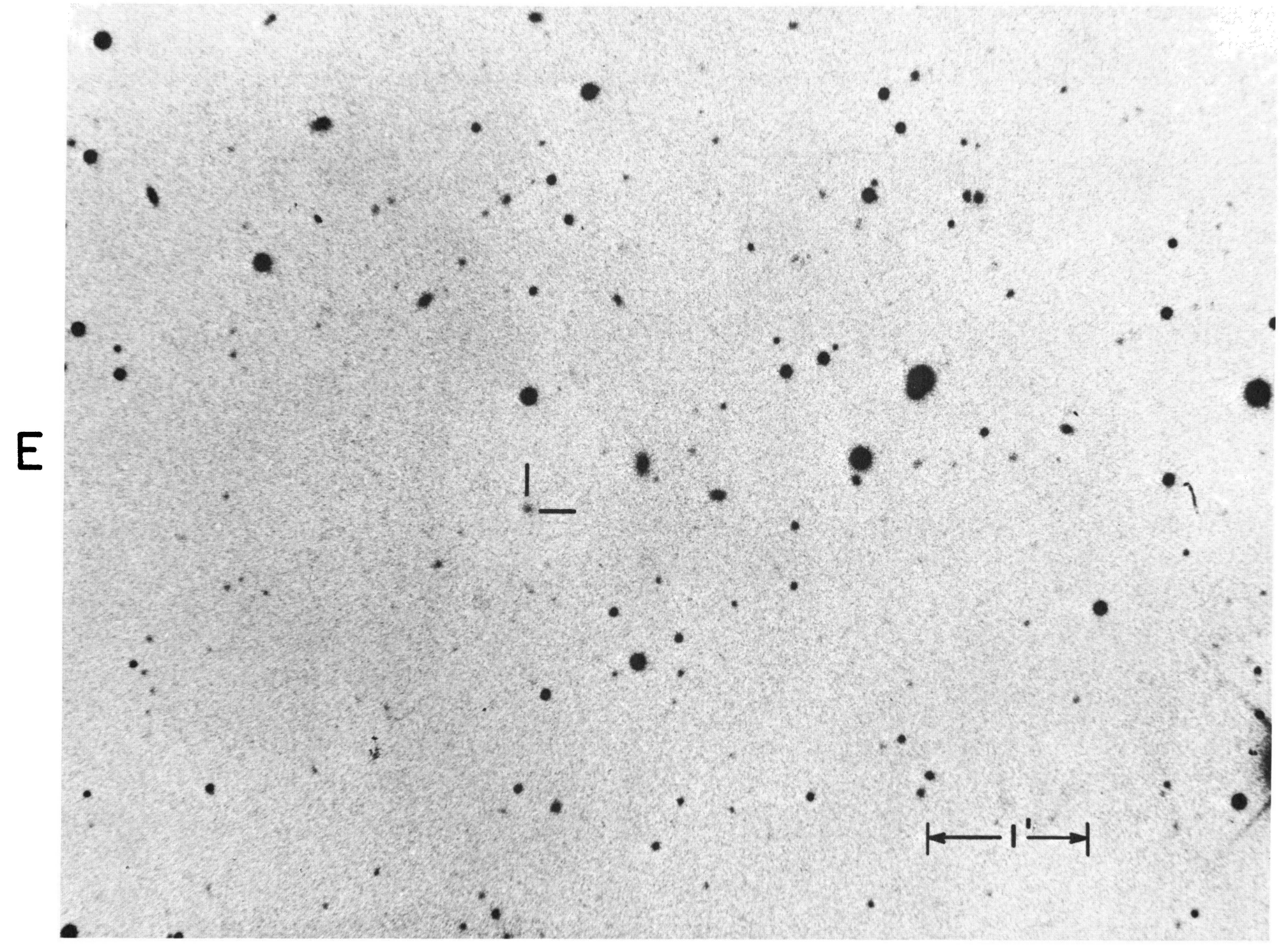

3C 343.1

Fig. 1.-An enlarged print from a $4 \mathrm{~m}$ reflector yellow-red direct plate of 3C 343.1. The exposure was on 127-04 emulsion behind a GG495 filter. The radio galaxy is marked; its 1950.0 optical position is: $\alpha=16^{\mathrm{h}} 37^{\mathrm{m}} 55 \mathrm{~s} 3, \delta=+62^{\circ} 40^{\prime} 34^{\prime \prime}$. 
!

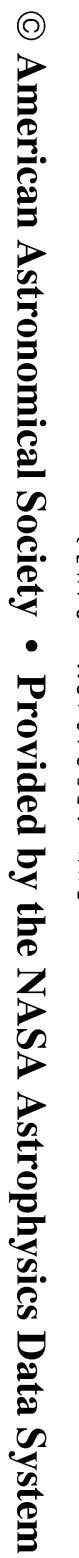

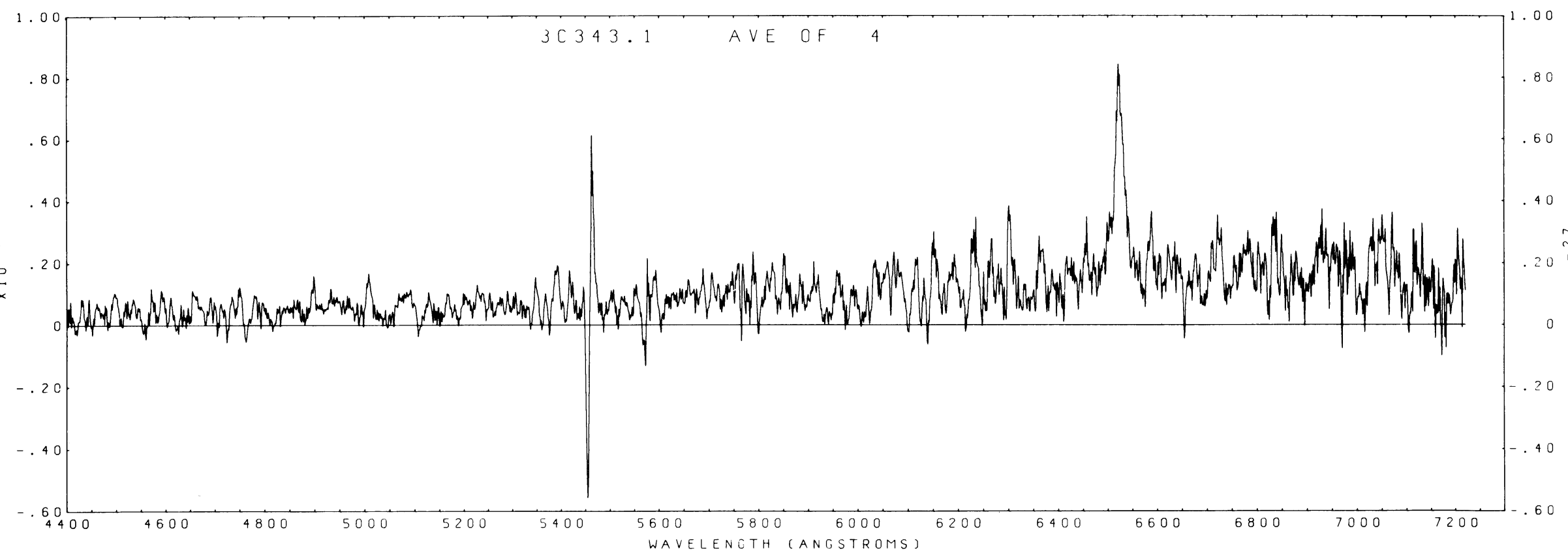

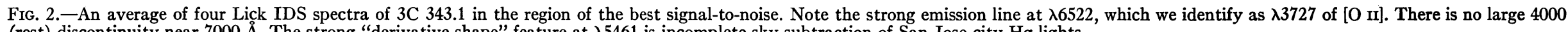
$\AA$ (rest) discontinuity near $7000 \AA$. The strong "derivative-shape" feature at $\lambda 5461$ is incomplete sky-subtraction of San Jose city Hg lights. 


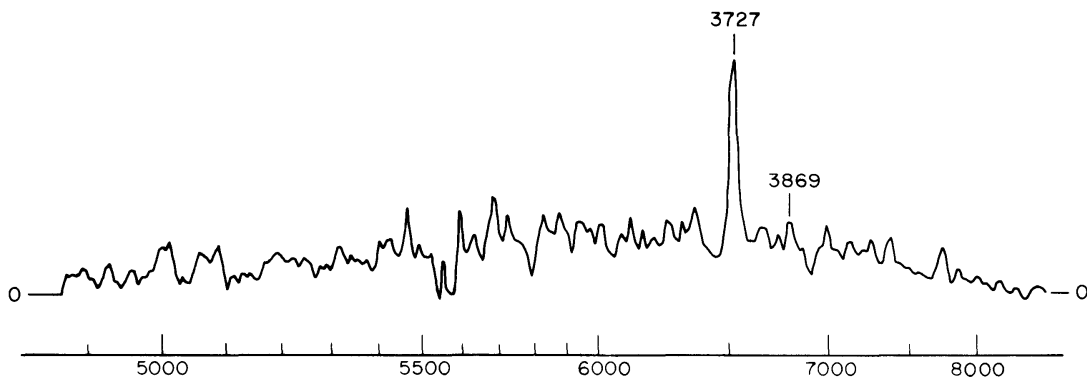

Fig. 3.-The Hale SIT vidicon spectrum of 3C 343.1, taken on 1976 June 5. Note the probable presence of [Ne III] $\lambda 3869$. Other "bumpy" features are not reproducible and are thought to be noise.

TABLE 2

\begin{tabular}{c} 
Photoelectric Photometry of \\
the $343,343.1$ Sources \\
\hline 3C 343.1 (galaxy, $z=0.750$ ): \\
$V=20.74 \pm 0.093$ \\
$R=19.65 \pm 0.049$ \\
$B=21.90 \pm 0.094$ \\
$V-R=1.09 \pm 0.105$ \\
$B-V=1.16 \pm 0.132$ \\
3C $343(\mathrm{QSO}, z=0.988):$ \\
$U=20.41 \pm 0.167$ \\
$B=21.06 \pm 0.085$ \\
$V=20.61 \pm 0.094$ \\
$R=19.55 \pm 0.035$ \\
$V-R=1.06 \pm 0.100$ \\
$B-V=0.45 \pm 0.127$ \\
$U-B=-0.65 \pm 0.187$ \\
\end{tabular}

cals, or (2) a strong nonthermal component may submerge all the starlight around an active nucleus in a radio galaxy.

Our present spectra cannot place useful direct limits on either mechanism; the signal-to-noise ratio is too poor to find with confidence individual Balmer $\mathrm{H}$ lines in absorption, although the Lick scans do show hints of $\mathrm{H} \delta$ and $\mathrm{H} \gamma$ absorption at 7179 and $7595 \AA$, respectively.

We would suggest that there are three indirect arguments against nonthermal (synchrotron, for example) optical light as the major continuum source in $3 \mathrm{C}$ 343.1.

First, well-observed single-emission-line galaxies rarely have strong nonstellar continua (Schmidt 1965; Costero and Osterbrock 1977). We estimate that on
Schmidt's visual emission intensity scale, 3C 343.1 would have a spectral activity index of $S_{6} \leq 3$. Schmidt's Table 2 suggests easy visibility of the underlying stellar continuum in this case.

Another indirect argument is that the galaxian volume observed with a 2 ".7 $\times 4^{\prime \prime}$ entrance slit is very large at $z=0.750$. With $H_{0}=50 \mathrm{~km} \mathrm{~s}^{-1} \mathrm{Mpc}^{-1}$ and $q_{0} \approx 0$, a metric rod of $10 \mathrm{kpc}$ length subtends $1^{\prime \prime}$. In nearby active galaxies, nonthermal continuum emission is generally restricted to the inner kiloparsec or less; hence, any nonthermal component would be swamped by the normal stellar continuum over the entrance aperture.

Finally, the position of 3C 343.1 on the red-magnitude Hubble diagram (Kristian, Sandage, and Westphal 1977 ) is not anomalous, compared to the extrapolated line through 3C 295 and 3C 330. This statement argues against extra (nonstellar) optical light in the 3C 343.1 spectrum.

In summary, we suggest that the blueness of $3 \mathrm{C} 343.1$ is due to the presence of rather early-type stars; an equivalent spectral type of about $\mathrm{F} 0 \mathrm{~V}$ would satisfy our very coarse present constraints. However, this suggestion of "recent" star formation, perhaps triggered by the radio outbursts itself, remains quite speculative.

Spinrad wishes to acknowledge the support of the NSF through grants AST 72-05061 and thanks John Stauffer for aid in observing and reductions. He also thanks Drs. Osterbrock and H. E. Smith for helpful discussions. Westphal and Kristian are supported in part by NSF grant GP-36450X.

\section{REFERENCES}

Baldwin, J., Burbidge, E. M., Hazard, C., Murdoch, H. S., Robinson, L. B., and Wampler, E. J. 1973, A p. J., 185, 739.

Costero, R., and Osterbrock, D. E. 1977, Ap. J., 211, 675.

Kellermann, K. I., Pauliny-Toth, I. I. K., and Williams, P. J. S. 1969, Ap. J., 157, 1.

Kristian, J., and Sandage, A. R. Ap.J., 162, 391.
Kristian, J., Sandage, A. R., and Westphal, J. 1977, Paris-IAU Colloquium No. 37 (in press).

Readhead, A. C. S., and Hewish, A. 1974, Mem. R.A.S., 78, 1. Schmidt, M. 1965, A p. J., 141, 1 .

Spinrad, H. 1976, Publ. A.S.P., 88, 565.

Jerome Kristian, Allan Sandage, and James Westphal: Hale Observatories, Carnegie Institution of Washington and California Institute of Technology, Pasadena CA 91125

Hyron SpINRAD: Department of Astronomy, University of California, Berkeley, CA 94720 